\title{
Attempted transverse impedance measurement in RHIC
}

\author{
M. Blaskiewicz \\ C. Montag
}

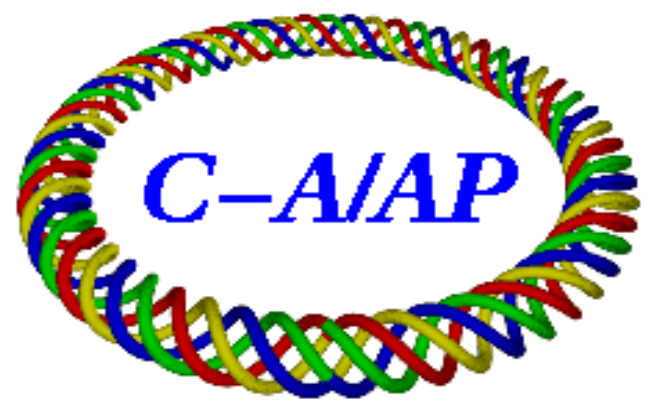

\section{Collider-Accelerator Department Brookhaven National Laboratory Upton, NY 11973}

Notice: This document has been authorized by employees of Brookhaven Science Associates, LLC under Contract No. DE-AC02-98CH10886 with the U.S. Department of Energy. The United States Government retains a nonexclusive, paid-up, irrevocable, world-wide license to publish or reproduce the published form of this document, or allow others to do so, for United States Government purposes. 


\title{
Attempted Transverse Impedance Measurement in RHIC
}

\author{
M. Blaskiewicz \\ C. Montag
}

July 20, 2012

\section{Results}

An attempt was made to improve earlier measurements of transverse impedance in $\mathrm{RHIC}^{1}$. Measurement with protons at injection were made on March 7, 2012. Data were taken in the yellow ring with the current as a function of time shown in Figure 1. We used the RHICBTF application with varying nummbers of bunches. The measured beam transfer functions are shown in Figures 2 and 3. The two indicies parameterizing each curve are the peak current and the number of bunches in the symmetric fill. For the horizontal data in Fig 1 the two blue curves for 8A peak current with 28 bunches have a larger measured tune difference than the green cure with $5 \mathrm{~A}$ peak current and 6 bunches or the magenta curve with $4 \mathrm{~A}$ peak current and 28 bunches. The expected tune shift for a transverse impedance of $4 \mathrm{M} \Omega / \mathrm{m}$ and 4 amps is 0.0012 . This is comparable to the measured vertical tune shift when changing from 8 to 28 bunches. The RHIC impedance is broadband so any change in tune with bunch number is an instrumental effect. We conclude that another measurement technique is needed to obtain reliable results.

\footnotetext{
1 "Transverse Impedance Measurements at the RHIC" S.Y. Zhang, H. Huang, P. Cameron, A. Drees, R. Fliller, T. Satogata, EPAC02, p1112, (2002)
} 


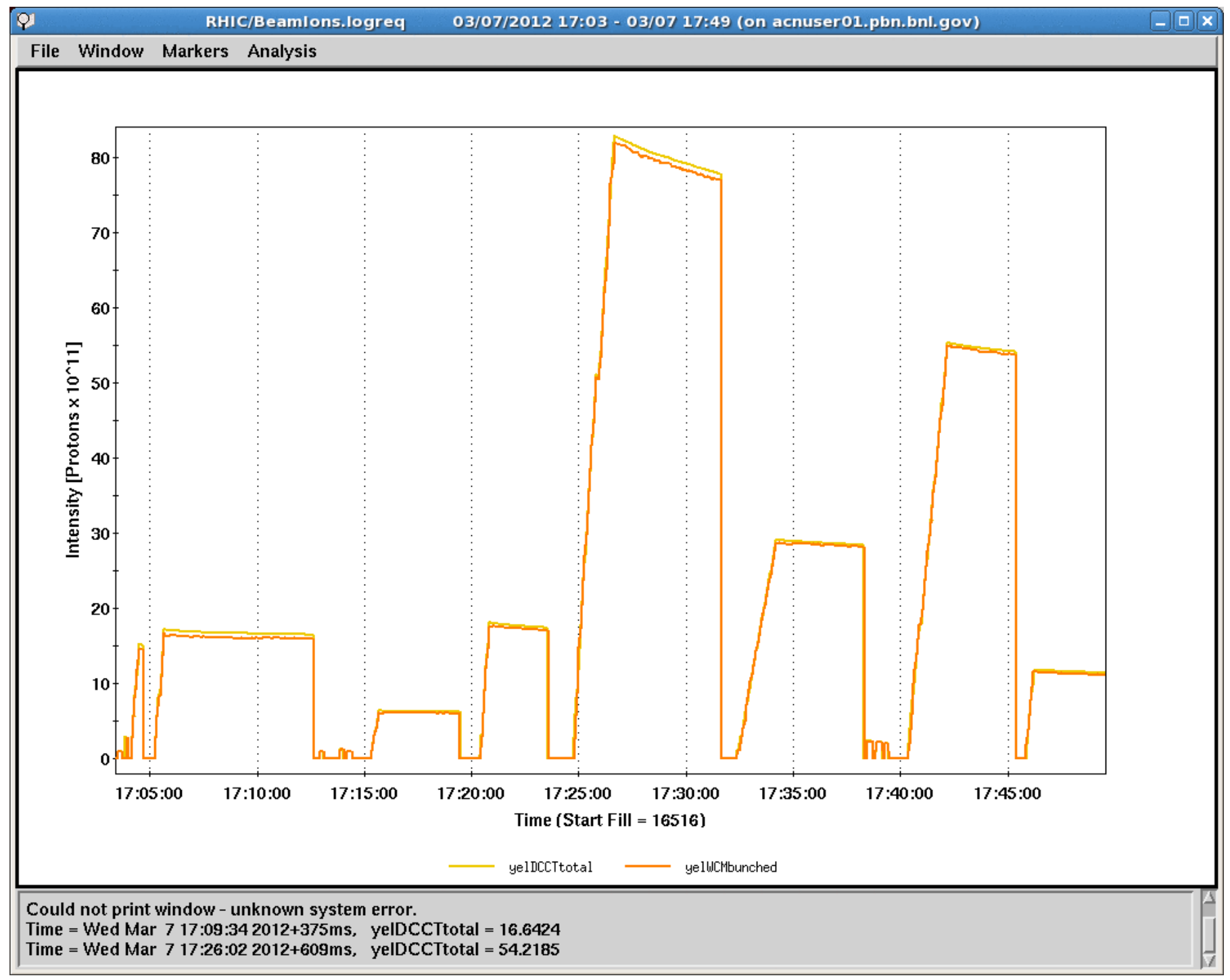

Figure 1: yellow beam current during the experiment 


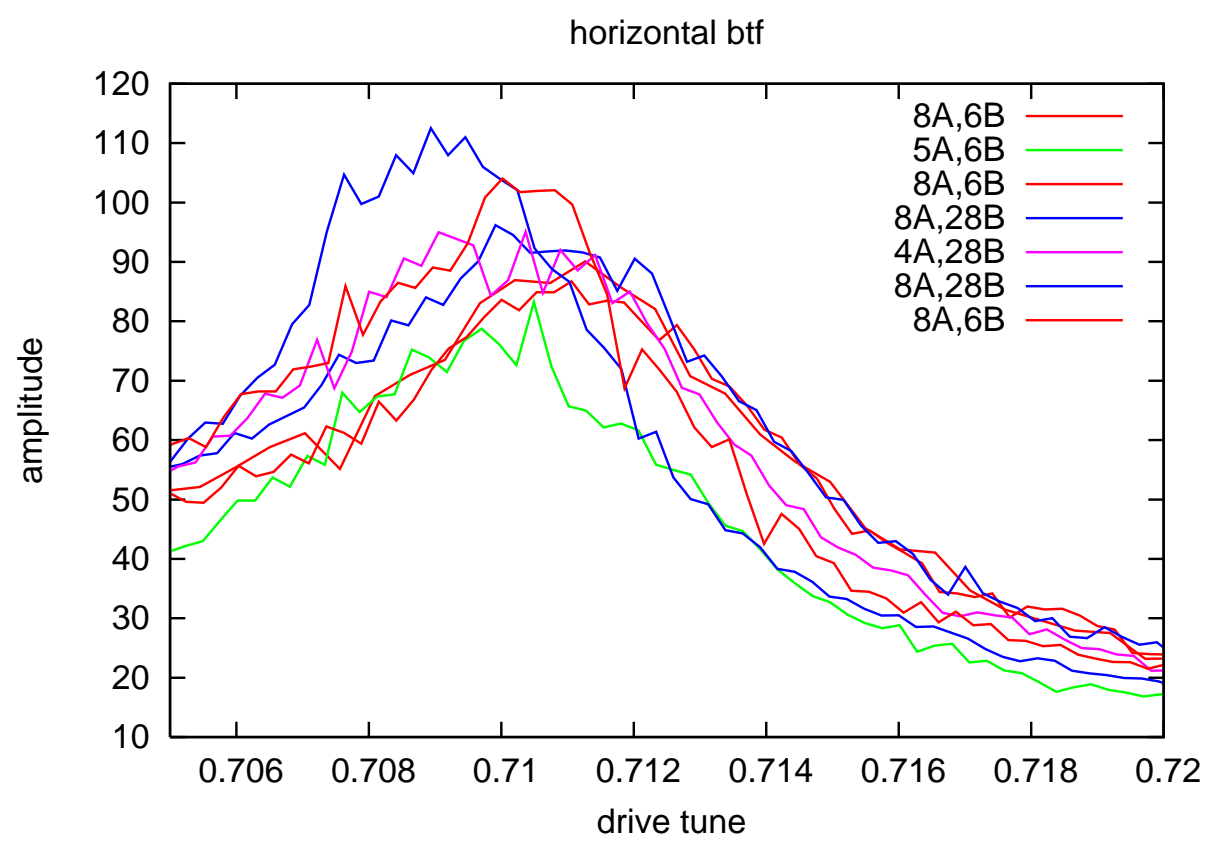

Figure 2: Horizontal BTFs for a range of intensities and fill patterns

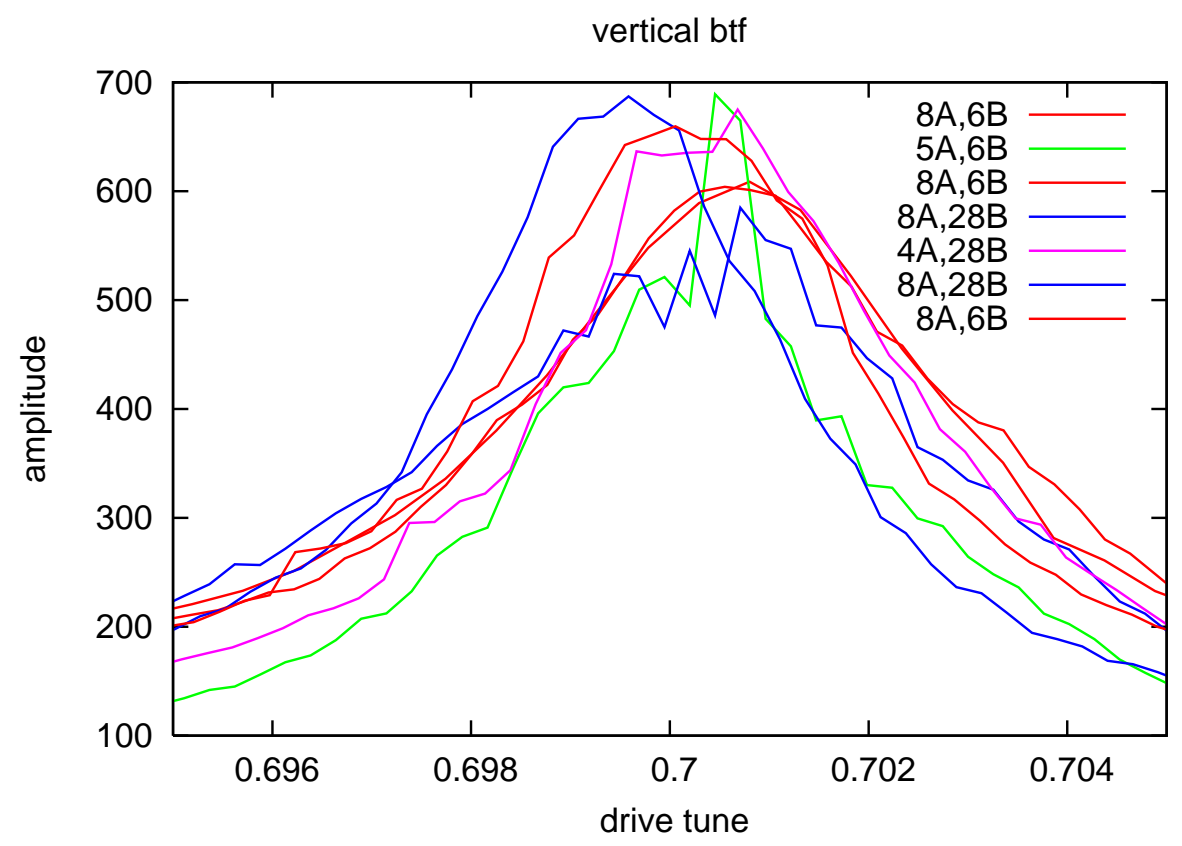

Figure 3: Vertical BTFs for a range of intensities and fill patterns 